\title{
Ricci dark energy in Chern-Simons modified gravity
}

\author{
J. G. Silva, A. F. Santos ${ }^{1}$ \\ ${ }^{1}$ Instituto de Física, Universidade Federal de Mato Grosso, \\ 78060-900, Cuiabá, Mato Grosso, Brazi*
}

\begin{abstract}
In this work, we have considered the Ricci dark energy model, where the energy density of the universe is proportional to the Ricci scalar curvature, in the dynamic Chern-Simons modified gravity. We show that in this context the evolution of the scale factor is similar to that displayed by the modified Chaplygin gas.
\end{abstract}

*Electronic address: jucelia,alesandroferreira@fisica.ufmt.br 
Currently the accelerated expansion of the universe has been strongly confirmed by some independent experiments such as the Cosmic Microwave Background Radiation (CMBR) [1] and Sloan Digital Sky Survey (SDSS) [2]. In an attempt to explain this phenomenon there are two possible paths; first option - propose corrections to general relativity, second option - assuming that there is a dominant component of the universe, a kind of antigravity called dark energy. Any way that we intend to follow, there are numerous models that attempt to explain this effect.

One of the models of modified gravity that has stood out in recent years is the ChernSimons modified gravity, which was initially developed in [3]. This modification consists in the addition of the Pontryagin density, which displays violation of parity symmetry in Einstein-Hilbert action. In four dimensions the Pontryagin density is simply a topological term, unless the coupling constant is not constant or promoted to a scalar field. The ChernSimons modification is not a random extension, but it is actually motivated by both string theory, as a necessary anomaly-canceling term to conserve unitarity [4], and loop quantum gravity [5]. For further details this proposal for a correction to general relativity, see the revision made in [6]. In this paper, we will study the dynamic formulation of Chern-Simons gravity, where the coupling constant is promoted to a scalar field, recent studies in this formulation include: neutron star binary [7], spacetime with and without black holes [8], among others.

From among the various models proposed for dark energy there are some that are based on the holographic principle, known as holographic dark energy [9] [10]. Such models are based on the idea that the energy density of a given system is proportional to the inverse square of some characteristic length of the system, for example, in [11] energy density is proportional to the Hubble scale, the future event horizon is used as the characteristic length in [9]. However, these models exhibit some fundamental problems such as the problem of fine tuning and/or coincidence problem. From these studies, it has been proposed in [12] a model of dark energy where the characteristic length is given by the average radius of the Ricci scalar, $R^{-1 / 2}$. Thus, the dark energy density is proportional to the Ricci scalar, i.e.,

$$
\rho_{x}=-\frac{\alpha}{16 \pi} R
$$

where $R$ is the Ricci scalar curvature and $\alpha$ is a constant to be determined. This model avoids the problems of fine tuning and coincidence that arise in previous models. It is 
a phenomenologically viable model and displays results similar to that presented by the cosmological model $\Lambda C D M$. In this work we will dedicate ourselves to investigate the Ricci dark energy in the context of Chern-Simons modified gravity for homogeneous and isotropic universe described by the Friedmann-Robertson-Walker (FRW) metric given by

$$
d s^{2}=-d t^{2}+a(t)^{2}\left(\frac{d r^{2}}{1-k r^{2}}+r^{2} d \theta^{2}+r^{2} \sin ^{2} \theta d \phi^{2}\right) .
$$

In this case the Ricci scalar curvature is given by

$$
R=-6\left(\dot{H}+2 H^{2}+\frac{k}{a^{2}}\right)
$$

where $k$ is the spatial curvature and $H=\frac{\dot{a}}{a}$ is the Hubble parameter. Then, the energy density becomes

$$
\rho_{x}=\frac{3 \alpha}{8 \pi}\left(\dot{H}+2 H^{2}+\frac{k}{a^{2}}\right) .
$$

Now let's study this dark energy model in the context of the dynamics Chern-Simons modified gravity. The action that describes this theory is given by

$$
S=\frac{1}{16 \pi G} \int d^{4} x\left[\sqrt{-g} R+\frac{l}{4} \theta^{*} R R-\frac{1}{2} \partial^{\mu} \theta \partial_{\mu} \theta+V(\theta)\right]+S_{\text {mat. }},
$$

where ${ }^{*} R R$ is the topological invariant called the Pontryagin term, $l$ is a coupling constant, the function $\theta$ is a dynamical variable, $S_{\text {mat }}$. is the action of matter and $V(\theta)$ is the potential, that for simplicity we equal to zero. Varying the action with respect to the metric $g_{\mu \nu}$ and to the scalar field $\theta$ we obtain the field equations, respectively

$$
\begin{gathered}
G_{\mu \nu}+l C_{\mu \nu}=8 \pi G T_{\mu \nu}, \\
g^{\mu \nu} \nabla_{\mu} \nabla_{\nu} \theta=-\frac{l}{64 \pi}^{*} R R,
\end{gathered}
$$

where $G_{\mu \nu}$ is the Einstein tensor and $C_{\mu \nu}$ is Cotton tensor defined by

$$
C^{\mu \nu}=-\frac{1}{2 \sqrt{-g}}\left[v_{\sigma} \epsilon^{\sigma \mu \alpha \beta} \nabla_{\alpha} R_{\beta}^{\nu}+\frac{1}{2} v_{\sigma \tau} \epsilon^{\sigma \nu \alpha \beta} R^{\tau \mu}{ }_{\alpha \beta}\right]+(\mu \longleftrightarrow \nu),
$$

with $v_{\sigma} \equiv \nabla_{\sigma} \theta, v_{\sigma \tau} \equiv \nabla_{\sigma} \nabla_{\tau} \theta$. The energy-momentum tensor is divided into two parts

$$
T_{\mu \nu}=T_{\mu \nu}^{R D E}+T_{\mu \nu}^{\theta}
$$

where

$$
T_{\mu \nu}^{\theta}=\left(\nabla_{\mu} \theta\right)\left(\nabla_{\nu} \theta\right)-\frac{1}{2} g_{\mu \nu}\left(\nabla_{\lambda} \theta\right)\left(\nabla^{\lambda} \theta\right)
$$


is the energy-momentum tensor associated with the scalar field and

$$
T_{\mu \nu}^{R D E}=\left(\rho_{x}+p_{x}\right) U_{\mu} U_{\nu}+p_{x} g_{\mu \nu}
$$

is the the energy-momentum tensor of the Ricci dark energy. We have that $U_{\mu}=(1,0,0,0)$ is the four-velocity, $\rho_{x}$ is the energy density (41) and $p_{x}$ is the pressure of dark energy.

Now let's study the component 00 of equation (6) which gives us the Friedmann equation

$$
G_{00}+C_{00}=8 \pi G\left(T_{00}^{R D E}+T_{00}^{\theta}\right)
$$

where

$$
\begin{aligned}
G_{00} & =3\left(\frac{\dot{a}^{2}}{a^{2}}+\frac{k}{a^{2}}\right), \\
T_{00}^{\theta} & =\frac{1}{2} \dot{\theta}^{2}, \\
T_{00}^{R D E} & =\rho_{x}=-\frac{\alpha}{16 \pi} R,
\end{aligned}
$$

and $C_{00}=0$. As discussed in [13] the components of the Cotton tensor vanish for all spherically symmetric metrics. Thus the Friedmann equation becomes

$$
\left(\frac{\dot{a}}{a}\right)^{2}+\frac{k}{a^{2}}=\alpha\left(\dot{H}+2 H^{2}+\frac{k}{a^{2}}\right)+\frac{4 \pi}{3} \dot{\theta}^{2},
$$

where we use natural units $c=G=1$. We have that $\dot{H}+2 H^{2}=\frac{\ddot{a}}{a}+\left(\frac{\dot{a}}{a}\right)^{2}$, thus

$$
\alpha \frac{\ddot{a}}{a}+\left(\frac{\dot{a}}{a}\right)^{2}(\alpha-1)+\frac{k}{a^{2}}(\alpha-1)+\frac{4 \pi}{3} \dot{\theta}^{2}=0 .
$$

Assuming a flat universe, $k=0$, we stay with

$$
\alpha \frac{\ddot{a}}{a}+(\alpha-1)\left(\frac{\dot{a}}{a}\right)^{2}+\frac{4 \pi}{3} \dot{\theta}^{2}=0 .
$$

Now let's look at the field equation associated with the scalar field (7). For the metric FRW we obtain that ${ }^{*} R R=0$, thus this equation becomes

$$
g^{\mu \nu} \nabla_{\mu} \nabla_{\nu} \theta=g^{\mu \nu}\left[\partial_{\mu} \partial_{\nu} \theta-\Gamma_{\mu \nu}^{\lambda} \partial_{\lambda} \theta\right]=0 .
$$

Choosing $\theta=\theta(t)$, as was done in [3] , this equation gives us

$$
\ddot{\theta}+3 \frac{\dot{a}}{a} \dot{\theta}=0
$$


Therefore, our set of field equations consists of the equations (18) and (20). Now our goal is to resolve them to determine the evolution of the scale factor $a(t)$. From the equation (20) we note that $\theta$ is related to $a(t)$ as

$$
\dot{\theta}=C a^{-3}
$$

where $C$ is a constant. Substituting this result into the equation (18) we obtain that

$$
\alpha \frac{\ddot{a}}{a}+(\alpha-1)\left(\frac{\dot{a}}{a}\right)^{2}+\beta a^{-6}=0,
$$

with $\beta \equiv \frac{4 \pi C^{2}}{3}$.

For simplicity we make the following change of variable

$$
u(a)=\frac{d a}{d t} \quad \Longrightarrow \quad u \frac{d u}{d a}=\frac{d^{2} a}{d t^{2}}
$$

Thus the equation (22) stays as

$$
\alpha u \frac{d u}{d a}+(\alpha-1) \frac{u^{2}}{a}+\beta a^{-5}=0 .
$$

Solving this equation for $u(a)$ we find

$$
u(a)=\frac{\sqrt{\beta+a^{2+\frac{2}{\alpha}}(1+\alpha) c_{1}}}{a^{2} \sqrt{1+\alpha}},
$$

where $c_{1}$ is an integration constant. Now returning to the original variables $a(t)$ and $t$ we can write

$$
t=\sqrt{1+\alpha} \int \frac{a^{2} d a}{\sqrt{\beta+a^{2+\frac{2}{\alpha}}(1+\alpha) c_{1}}} .
$$

Solving this integral for $a(t)$ we find that

$$
t=\xi a_{2}^{3} F_{1}\left[\frac{1}{2}, \frac{3 \alpha}{2 \zeta} ; 1+\frac{3 \alpha}{2 \zeta} ;-\frac{a^{2+\frac{2}{\alpha}} c_{1} \zeta}{\beta}\right],
$$

where ${ }_{2} F_{1}$ is the hypergeometric function, $\zeta=1+\alpha$ and $\xi=\frac{1}{3} \sqrt{\frac{\zeta}{\beta}}$. At this time, let's analyze this result assuming $\alpha \approx \frac{1}{2}$ as found in [12]. Thus

$$
t=\frac{a^{3}}{\sqrt{6 \beta}}{ }_{2} F_{1}\left[\frac{1}{2}, \frac{1}{2} ; \frac{3}{2} ;-\frac{3 c_{1}}{2 \beta} a^{6}\right] .
$$


This result can be rewritten as

$$
a^{\frac{3(1+A)}{2}}{ }_{2} F_{1}\left[x, x ; 1+x ;-\frac{B}{C(1+A)} a^{\frac{3(1+A)}{2 x}}\right]=\frac{\sqrt{3}}{2}(1+A) C^{x} t,
$$

where $A=1, B=6 c_{1}, C=2 \beta$ and $x=\frac{1}{2}$.

We can also analyze this result (28) by writing the hypergeometric function as a hypergeometric series [14]. Doing this, we see that this series is equal to series of the function arcsinh and so we can write

$$
t=\frac{1}{3 \sqrt{c_{1}}} \operatorname{arcsinh}\left[\sqrt{\frac{3 \mathrm{c}_{1}}{2 \beta}} \mathrm{a}^{3}\right] .
$$

In this way, the scale factor can be written as

$$
a(t)=\left(\frac{2 \beta}{3 c_{1}}\right)^{\frac{1}{6}} \sinh ^{\frac{1}{3}}\left(3 \sqrt{c_{1}} t\right) .
$$

Comparing the result (29) with the developments in [15] we observe that they are similar. Can also be seen here that the result for the scale factor, equation (31), is similar to those obtained in [16] and [17].

Therefore, we conclude that we find for suitable choices of some parameters that the Ricci dark energy model in the Chern-Simons gravity displays the same results obtained from the modified Chaplygin gas, i.e., our results show that there is a correspondence between the Ricci dark energy in gravity Chern-Simons and the modified Chaplygin gas. This correspondence between the Ricci dark energy model and the Chaplygin gas has also already been obtained in other contexts as shown in [18] [19]. Therefore, in our case, we found a gravity model that exhibits the same behavior displayed by the modified Chaplygin gas.

\section{Acknowledgments}

This work was supported by the Project FAPEMAT/CNPq No. 685524/2010. J. G. Silva thanks Coordenação de Aperfeiçoamento de Pessoal de Nível Superior (CAPES) for financial support.

[1] D. N. Spergel, et. al., Astrophys. J. Suppl. 170, (2007) 377, arXiv:astro-ph/0603449. 
[2] J. K. Adelman-McCarthy, et. al., Astrophys. J. Suppl. 175, (2008) 297, arXiv:0707.3413 astro$\mathrm{ph}]$.

[3] R. Jackiw and S. Y. Pi, Phys. Rev. D 68, (2003) 104012, arXiv:gr-qc/0308071.

[4] J. Polchinski, Superstring Theory and Beyond String Theory vol.2 (Cambridge University Press, Cambridge, UK, 1998).

[5] A. Ashtekar, A.P. Balachandran, and S. Jo, Int. J. Mod. Phys. A 4, (1989) 1493.

[6] S. Alexander and N. Yunes, Phys. Rep. 480, (2009) 155, arXiv:0907.2562[hep-th].

[7] K. Yagi, L. C. Stein, N. Yunes and Takahiro Tanaka, arXiv:1302.1918[gr-qc].

[8] T. Shiromizu, K. Tanabe, arXiv:1303.6056 [gr-qc].

[9] M. Li, Phys. Lett. B 603, (2004) 1, arXiv:hep-th/0403127.

[10] S. D. H. Hsu, Phys. Lett. B 594, (2004) 13, arXiv:hep-th/0403052.

[11] A. Cohen, D. Kaplan and A. Nelson, Phys. Rev. Lett. 82, (1999) 4971, arXiv:hep-th/9803132.

[12] C. Gao, F. Wu, X. Chen and Y-G. Shen, Phys. Rev. D 79, (2009) 043511, arXiv:0712.1394[astro-ph].

[13] D. Grumiller and N. Yunes, Phys. Rev. D. 77, (2008) 044015, arXiv:0711.1868[gr-qc].

[14] I. S. Gradshteyn and I. M. Ryzhik, Table of Integrals, Series and Products (Academic Press, 2000).

[15] U. Debnath, A. Banerjee and S. Chakraborty, Class. Quant. Grav. 21, (2004) 5609, arXiv:gr-qc/0411015.

[16] S. S. Costa, Mod. Phys. Lett. A 24, (2009) 531, arXiv: 0803.4006[gr-qc].

[17] J. C. Fabris, S. V. B. Goncalves, R. de Sa Ribeiro, Gen. Rel. Grav. 36, (2004) 211, arXiv: astro-ph/0307028

[18] L. P. Chimento and M. G. Richarte, Phys. Rev. D 84, (2011) 123507, arXiv:1107.4816[astroph.CO].

[19] A. Pasqua, M. Jamil, R. Myrzakulov and B. Majeed, Phys. Scripta 86, (2012) 04, arXiv:1211.0902[physics.gen-ph]. 